\title{
CONNECTIVITY PROPERTIES OF RANDOM WAYPOINT MOBILITY MODEL FOR AD HOC NETWORKS*
}

\author{
Pasi Lassila, Esa Hyytiä, and Henri Koskinen \\ Helsinki University of Technology (TKK), Networking Laboratory \\ P.O. Box 3000, FIN-02015 TKK, Finland \\ \{pasi.lassila, esa.hyytia,henri.koskinen\}@tkk.fi
}

\begin{abstract}
We study the connectivity properties of an ad hoc network consisting of $n$ nodes each moving according to the Random Waypoint mobility model. In particular, we focus on estimating two quantities, the probability that the network is connected, and the mean durations of the connectivity periods. The accuracy of the approximations is compared against numerical simulations. For the probability of connectivity, an approximation is given that is remarkably accurate. By numerical examples we also show that in sparse network the mobility has a positive effect on connectivity, whereas in dense network the situation becomes the opposite. For the mean length of the connectivity periods results are also accurate in the important region where the probability of connectivity rises rapidly.
\end{abstract}

Keywords: ad hoc networks, mobility modelling, $k$-connectivity, RWP.

\section{Introduction}

The connectivity problem in wireless networks deals with determining if it is possible to transfer information between any two nodes, typically ignoring all capacity and traffic related phenomena, most notably interference effects. The most popular network model - and the one used in this study - defining when two nodes are directly connected has been the Boolean one, in which two nodes are connected if they are both within each other's transmission ranges. When this model is augmented with an assumption that all nodes have an equal transmission range, the connectivity problem reduces to determining the distribution of the threshold range for connectivity: for a given set of nodes, this is equal to the greatest edge length in the minimum spanning tree of the nodes [6]. It has been shown in [7] that for uniformly distributed nodes in the unit

* We are grateful to Laura Nieminen for the help with the simulations. This work has been supported by the Academy of Finland (grant n:o 74524), the Finnish Defence Forces Technical Research Centre and partly the Nokia Foundation. 
square, as the number of nodes tends to infinity, the threshold range for connectivity has asymptotically the same, previously known, distribution as the threshold range for minimum degree 1, i.e., the greatest edge length in the nearest-neighbor graph. The result has been generalized to $k$-connectivity in [8]. Furthermore, the identity in the case $k=1$ has been shown to hold for normally distributed points in [9]. Recently, the asymptotic distributions of the threshold range for $k$-connectivity when $k>1$, for uniformly distributed points inside a circle and square have been derived in [10]. The distribution of the threshold range for $k$-connectivity is not known when the number of nodes is finite. The results above motivate approximating $k$-connectivity of finite networks by minimum degree $k$, as has been done, e.g., in [11]; this is also the basis of our approach.

In this paper we present approximations for the probability that a network with $n$ nodes is $k$-connected. The network nodes are assumed to move according to random waypoint (RWP) mobility model [1-4], which concentrates more nodes in the center of the area. In particular, for our purposes an important quantity is the stationary node distribution. For this approximate results for various movement areas (circle, rectangle) have been obtained in [1,3], and, as a part of our earlier work, in [4] we have also derived an exact expression for an arbitrary convex domain. In the RWP model the nodes move independently and the number of neighbors a given node has is binomially distributed with a certain parameter $p$. These are needed in our approximation for the probability that all nodes have at least $k$ neighbors, which is used to approximate the probability of $k$-connectivity. In our first approximation the parameter $p$ is computed exactly using the results of our earlier work [4]. Additionally, two numerically simpler approximation schemes are given, which are based on making some additional poissonian assumptions. Our approach is similar to the one in [11], with the distinction that in [11] the binomial distribution characterizing the number of neighbors a given node has is approximated by a Poisson distribution. Also, we have an exact result for the node distribution, whereas in [11] an approximation has been used (although a rather accurate one). The quality of the approximations for 1-, 2- and 3-connectivity are evaluated by means of numerical simulations in a unit disk, while the approach itself is not limited to any special geometry. In the simulations, the threshold ranges for $k$-connectivity have been determined using the efficient algorithms given in [12]. The results show that especially our first approximation gives remarkably accurate results. We also give an approximation for the mean time a network remains 1-connected (or disconnected). The approximation utilizes the results on the node arrival rate into a given subset given in [5]. The numerical simulations show that the approximation gives reasonably accurate estimates in the most important region where the probability of connectivity rises rapidly. 


\section{Preliminaries}

In the RWP model, a node moves, independently of the others, directly towards the next waypoint at a certain velocity $v$ in a convex domain denoted by $\mathcal{A}$. Upon reaching the waypoint, the next waypoint and velocity are drawn randomly from the uniform distribution over $\mathcal{A}$ and the velocity distribution, respectively. Next we state the necessary results from $[4,5]$ for our purposes. Let $\bar{\ell}$ denote the mean length of a leg and $A$ the area of the domain $\mathcal{A}$. Define

$$
h(\mathbf{r}, \phi)=\frac{1}{2} \cdot a_{1} a_{2}\left(a_{1}+a_{2}\right),
$$

where $a_{1}=a_{1}(\mathbf{r}, \phi)$ denotes the distance from $\mathbf{r} \in \mathcal{A}$ to the border of $\mathcal{A}$ in direction $\phi$, and $a_{2}$ denotes the distance to the border in the opposite direction. The stationary distribution of an RWP node is given by (see [4])

$$
f(\mathbf{r})=\frac{1}{C} \int_{0}^{2 \pi} h(\mathbf{r}, \phi) d \phi=\frac{h(\mathbf{r})}{C}, \quad \text { where } C=\bar{\ell} A^{2} .
$$

The mean arrival rate into a subset $\mathcal{A}_{j} \subset \mathcal{A}$ is given by [5]

$$
\lambda\left(\mathcal{A}_{j}\right)=\int_{\partial \mathcal{A}_{j}} \lambda(\mathbf{r}, \theta(d \mathbf{r})) d r
$$

where $\theta(d \mathbf{r})$ is the direction of the tangent at point $\mathbf{r}$, and

$$
\lambda(\mathbf{r}, \theta)=\frac{1}{C \cdot \mathrm{E}[1 / v]} \int_{0}^{\pi} \sin \phi \cdot h(\mathbf{r}, \theta+\phi) d \phi .
$$

For unit disk the pdf of node location, denoted by $f(r)$ with $r=|\mathbf{r}|$, is given by

$$
f(r)=\frac{2\left(1-r^{2}\right)}{C} \int_{0}^{\pi} \sqrt{1-r^{2} \cos \phi} d \phi,
$$

where $C=128 \pi / 45 \approx 8.936$ [4]. Let $\lambda(r, d)$ denote the mean arrival rate into a disk with a radius of $d$ located $r$ units from the origin. Using (2) gives

$$
\lambda(r, d)=\frac{45 / 64}{\pi \mathrm{E}[1 / v]} \int_{\alpha_{0}}^{\pi} d \alpha d\left(1-x^{2}\right) \int_{0}^{\pi} d \phi \sin \phi \sqrt{1-x^{2} \cos ^{2}(\phi+\alpha-\beta)}
$$

where $x^{2}=r^{2}+2 r d \cos \alpha+d^{2}, \beta=\arctan (r+d \cos \alpha, d \sin \alpha)$, and

$$
\alpha_{0}= \begin{cases}0, & \text { when } r+d<1, \\ \arccos \frac{1-r^{2}-d^{2}}{2 r d}, & \text { when } r-d<1 \leq r+d, \\ \pi & \text { otherwise. }\end{cases}
$$

For the special case $r=0$ we have $x=d, \alpha_{0}=0$ and $\alpha=\beta$ yielding

$$
\lambda(d)=\frac{45 \cdot d\left(1-d^{2}\right)}{64 \cdot E[1 / v]} \int_{0}^{\pi} \sin \phi \cdot \sqrt{1-d^{2} \cos ^{2} \phi} d \phi .
$$




\section{Analytical Approximations for Connectivity}

We study $k$-connectivity and focus on the case where the movement of the nodes is restricted to a unit disk. In particular, we are interested in the probability that a network with $n$ nodes is $k$-connected at an arbitrary point of time and denote this by $C_{n, k}(d)$, where $d$ is the transmission range. A network is said to be (1-)connected if there exists a path between all node pairs, and $k$-connected if for each node pair at least $k$ node disjoint paths exist. Due to the assumed circular shape, the node distribution depends only on the distance $r=|\mathbf{r}|$ from the center, as given by (3). The coverage area of each node is also assumed to be circular with a radius of $d$ and is denoted by $B_{d}(\mathbf{r})$. Note that in principle, the domain of movement can be any convex region, and our general result (1) on the pdf $f(\cdot)$ holds. The approximations presented below depend on the shape of the domain through $f(\cdot)$ and thus hold for any convex region.

Approximation 1: Denote by $Q_{n, k}(d)$ the probability that an arbitrary node has at least $k$ neighbors. Consider an arbitrarily chosen node and condition on its location, denoted by $\mathbf{r}$. Let $p(r, d)$ denote the probability that a given node is within $B_{d}(\mathbf{r})$, where we emphasize that this probability depends only on the distance $r=|\mathbf{r}|$ from the center. We can express $p(r, d)$ as

$$
p(r, d)=\int_{\mathbf{x} \in B_{d}(\mathbf{r})} f(|\mathbf{x}|) d A
$$

where $\mathrm{x}$ denotes the vector for the location of a point inside $B_{d}(\mathbf{r})$. With a probability of $1-p(r, d)$ the arbitrary node is outside $B_{d}(\mathbf{r})$. Since all nodes are independent, the number of other nodes within domain $B_{d}(\mathbf{r})$ obeys a binomial distribution, $N_{r, d} \sim \operatorname{Bin}(n-1, p(r, d))$, and the probability that a given node is connected to at least $k$ nodes equals

$$
1-\sum_{i=0}^{k-1}\left(\begin{array}{c}
n-1 \\
i
\end{array}\right) \cdot p(r, d)^{i} \cdot(1-p(r, d))^{n-1-i} .
$$

With the RWP model in unit disk the probability density that a node is at a distance $r$ from the center is $2 \pi r f(r)$, and $Q_{n, k}(d)$ is given by

$$
Q_{n, k}(d)=2 \pi \int_{0}^{1} r f(r)\left(1-\sum_{i=0}^{k-1}\left(\begin{array}{c}
n-1 \\
i
\end{array}\right) p(r, d)^{i}(1-p(r, d))^{n-1-i}\right) d r
$$

which is an exact result. As in [11], we approximate $k$-connectivity by

$$
C_{n, k}(d)=\mathrm{P}\{n \text { nodes are } k \text {-connected }\} \approx\left(Q_{n, k}(d)\right)^{n} .
$$

Note that for $n=2$ and $k=1$ one should use the exact result $C_{2,1}(d)=Q_{2,1}(d)$ given by (6) instead. 
The formal motivation of this approximation is as follows. As remarked in [7], for uniformly distributed random points, the asymptotics of the greatest edge length in the nearest neighbor graph are as if the nearest-neighbor distances were independent, and the longest edge is likely to be the same for the nearest neighbor graph and the minimum spanning tree. Because this holds for normally distributed points [9], the same can be expected to hold for more general spatial distributions. Here, we make the additional assumption that this generalizes to $k$-connectivity and the $k$-nearest neighbor graph. Note that $Q_{n, k}(d)$ can, as a function of $d$, be interpreted as the cumulative distribution function of a single $k$-nearest-neighbor distance. Hence $\left(Q_{n, k}(d)\right)^{n}$ is the cumulative distribution of the maximum of $n$ such i.i.d. $k$-nearest-neighbor distances, and by the above, this is approximated to be the distribution for the greatest $k$-nearest-neighbor distance. The final approximation then sets this distribution equal to that of the threshold range for $k$-connectivity.

Approximation 2: A more simple approximation can be developed by also making an approximation in computing the probability that a certain number of nodes exist within the coverage area of a given node. More specifically, we make a local Poisson assumption and assume that the nodes within the coverage area $B_{d}(\mathbf{r})$ result from a homogeneous Poisson point process with intensity $\lambda=f(\mathbf{r})$, i.e., the number of nodes within $B_{d}(\mathbf{r})$ obeys a Poisson distribution with mean equal to $\lambda$ times the area of $B_{d}(\mathbf{r})$.

Similarly as in the case of Approximation 1, we condition on the location of a single node, and have a superposition of $n-1$ identical Poisson point processes yielding a total intensity of $(n-1) \cdot f(\mathbf{r})$ per unit area. Consequently, the number of nodes residing in $B_{d}(\mathbf{r})$ obeys a Poisson distribution with mean

$$
a(r)=(n-1) \pi d^{2} \cdot f(r),
$$

and the probability that the number of nodes within $B_{d}(\mathbf{r})$ is less than $k$ is given by $\sum_{i=0}^{k-1} \frac{a(r)^{i}}{i !} e^{-a(r)}$. Thus, our approximatation for $Q_{n, k}(d)$ is

$$
\hat{Q}_{n, k}(d)=1-2 \pi \int_{0}^{1} r f(r) \sum_{i=0}^{k-1} \frac{a(r)^{i}}{i !} \cdot e^{-a(r)} d r .
$$

In the above, it is assumed that the coverage area is a full circle even on the border of the RWP domain. The limiting effect of the border can be taken into account by introducing a function $A(r, d)$ which gives the area of the intersection of the unit disk and a disk with radius $d$ at a distance of $r$ from the origin, $A(r, d)=\left|B_{1}(0) \cap B_{d}(r)\right|$. With this notation the slightly more accurate approximation for $a(r)$ can be expressed as

$$
a(r)=(n-1) \cdot A(r, d) \cdot f(r) .
$$

Finally, we use the same approximation for the probability of $k$-connectivity as in (7), i.e., $C_{n, k}(d) \approx\left(\hat{Q}_{n, k}(d)\right)^{n}$. 


\section{Length of Connectivity Periods}

Another important performance measure is the mean time the network remains connected. Let random variables $T_{c}$ and $T_{d}$ denote the lengths of the time periods the network is connected and disconnected, respectively. Clearly,

$$
C_{n, 1}(d)=\mathrm{P}\{n \text { nodes are 1-connected }\}=\frac{\bar{T}_{c}}{\bar{T}_{c}+\bar{T}_{d}} .
$$

As we are interested in $\bar{T}_{c}$, knowledge of $C_{n, 1}(d)$ and $\bar{T}_{d}$ is sufficient. For small values of $d$ the network is disconnected with high probability, but as $d$ increases beyond a critical value (depends on $n$ ) the probability of connectivity starts to increase rapidly. In practise, this is perhaps the most interesting region, where, when $n$ is large, typically only one node is separated from the network when the network becomes disconnected. We propose estimating $\bar{T}_{d}$ by the mean interarrival time of nodes into a disk $B_{d}(\mathbf{r})$ (radius $d$, center $r$ units away from the origin). Recall that, $\lambda(r, d)$ denotes the arrival rate of a single node into a disk $B_{d}(\mathbf{r})$, and using either (4) or (5), as the case may be, one can compute $\lambda(r, d)$. Let $\bar{T}_{d}^{(r)}$ denote the mean disconnectivity time on condition that a single node gets isolated at point $r$, which we can estimate by

$$
\bar{T}_{d}^{(r)} \approx \hat{T}_{d}^{(r)}=\frac{1}{(n-1) \cdot \lambda(r, d)}
$$

Next we approximate $\bar{T}_{d}$ by $\hat{T}_{d}^{(r)}$ with some $r$,

$$
\bar{T}_{d} \approx \hat{T}_{d}^{(r)}
$$

or in general case by the integral

$$
\bar{T}_{d} \approx \int_{r} T_{d}^{(r)} \cdot g(r) d r
$$

where $g(r)$ corresponds to the probability that the isolated node is located at the distance of $r$ from the origin. Note that in (12) and (13) we assume that disconnectivity is due to one isolated node. In (12) we are parameterizing the approximation with respect to the distance $r$ from the center, and in the numerical experiments we use $r=0$ and $r=1$, which imply that we assume that the network network typically becomes disconnected when a single node gets isolated either at the center $(r=0)$ or on the border $(r=1)$. In (13) we assume some distribution for the location of the isolated node, and in the numerical experiments we use the uniform distribution, $g(r)=2 \pi r(1 / \pi)=$ $2 r$. Finally, combining the above with (7) gives us an estimate for $\bar{T}_{c}$ :

$$
\bar{T}_{c}=\frac{C_{n, 1}(d)}{1-C_{n, 1}(d)} \bar{T}_{d} \approx \frac{p^{n}}{1-p^{n}} \cdot \hat{T}_{d},
$$

where $p$ denotes the probability that a node has at least one neighbor, $p=Q_{n, 1}(d)$. 

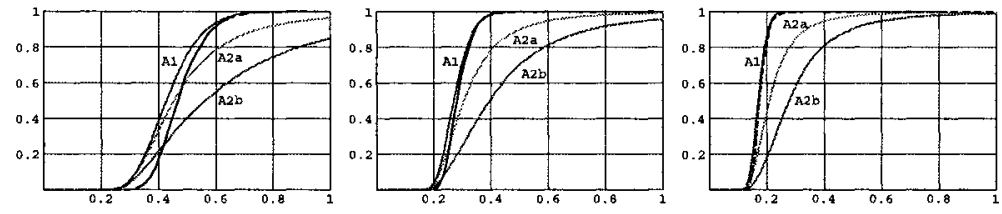

Figure 1. Validation of 1-connectivity for $n=20,100,500$ nodes (from left to right) as a function of $d$, dashed lines depict simulations and solid lines analytical results.
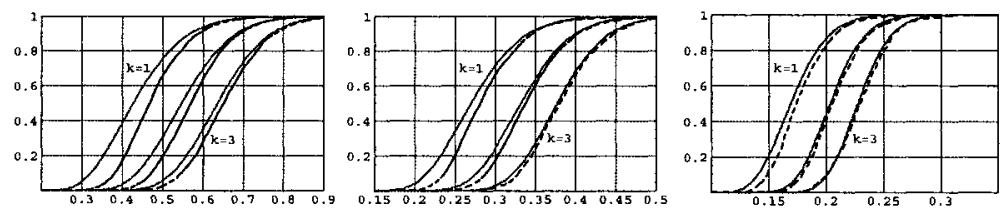

Figure 2. Validation of k-connectivity for $n=20,100,500$ nodes (from left to right) as a function of $d$, dashed lines depict simulations and solid lines analytical results.

\section{Numerical Examples}

\section{Probability of Connectivity}

First we compare the accuracy of the approximations for 1-connectivity for the different number of nodes $n$. We refer as A1 to Approximation 1. Approximation 2 contains two approximations and they are referred to as $A 2 a$ and $A 2 b$, where A2a refers to the approximation with $a(r)$ given by (8), i.e., the domain $B_{d}(\mathbf{r})$ is a full circle even at the border, and $\mathrm{A} 2 \mathrm{~b}$ refers to the approximation with $a(r)$ given by $(10)$, i.e., the "border effect" is taken into account. The results are shown in Fig. 1, where the dashed lines correspond to simulated results and solid lines to the approximations (as indicated in the figures). As can be seen, A1 is remarkably accurate as $n$ increases. Also, both A2a and A2b are able to predict well the initial rise, but they do not rise as steeply as they should. Somewhat surprisingly, the more detailed approximation A2b which includes the proper handling of the border effect, is less accurate than the simpler A2a.

The results for 2- and 3-connectivity are shown in Fig. 2, where in each graph we show simultaneously 1-, 2- and 3-connectivity as a function of $d$. In the simulations the $k$-connectivity has been determined using the algorithms from [12]. The results only compare the accuracy of A1 (solid lines) to simulated results (dashed lines) as the accuracy of $\mathrm{A} 2 \mathrm{a}$ and $\mathrm{A} 2 \mathrm{~b}$ is similar to that already shown before. It can be seen that Al is very close to the simulated values as $n$ increases, and the higher the value of $k$ the better the fit.

Finally, we compare A1 with the approximation given in [11], where the aim has been to study connectivity in large networks. Fig. 3 shows the results of 1-connectivity for A1 (solid lines), the approximation from [11] (dotted lines) 

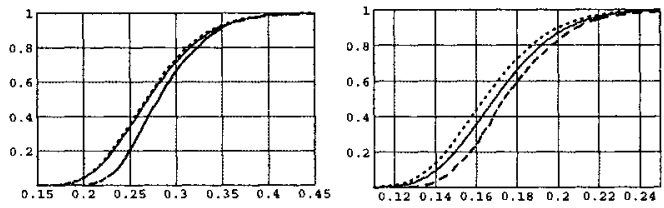

Figure 3. Comparison of the accuracy of A1 (solid lines) and the approximation in [11] (dotted lines) against simulations (dashed lines) for $n=100,500$ (left, right) nodes.
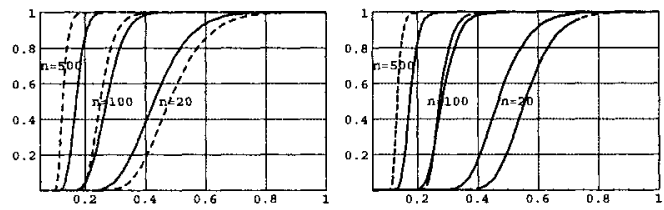

Figure 4. Comparisons for $C_{n, 1}(d)$ with RWP node distribution (solid lines) and uniform node distribution (dashed lines) using our approximations (left) and simulations (right).

and simulations (dashed lines) for $n=100$ nodes (left figure) and $n=500$ nodes (right figure). As can be seen, $\mathrm{A} 1$ is more accurate, especially for $n=500$.

Next we compare the impact on 1-connectivity of a uniform vs. the RWP node location distribution. The analytical results for the RWP case correspond to approximation $\mathrm{A} 1$, and the results for the uniform case are obtained from A1 by using $f(r)=1 / \pi$. The results are shown in Fig. 4, where the figure on the left contains results obtained by using our analytical approximations, and the figure on the right contains the corresponding simulated results. Each figure depicts $C_{n, 1}(d)$ as a function of $d$ for $n=20,100,500$. Solid lines correspond to connectivity under RWP node distribution and dashed lines to connectivity under uniform node distribution. It can be seen that the mobility induced by the RWP model can either improve or degrade the connectivity probability depending on the number of nodes. In particular, for small number of nodes, connectivity properties gain from mobility. However, as the number of nodes is increased, the situation becomes the opposite. This phenomenon occurring in the simulations is also captured by our analytical approximations although numerical accuracy is not perfect for small number of nodes.

\section{Mean Length of Connectivity Periods}

In Fig. 5 the estimated mean lengths of the connectivity periods are depicted as a function of $d$ and compared against simulations, when the speed is constant, $v=1$, and the number of nodes $n=20,100,500$. Simulation results are indicated with dashed lines and triangle markers. Lines with square 

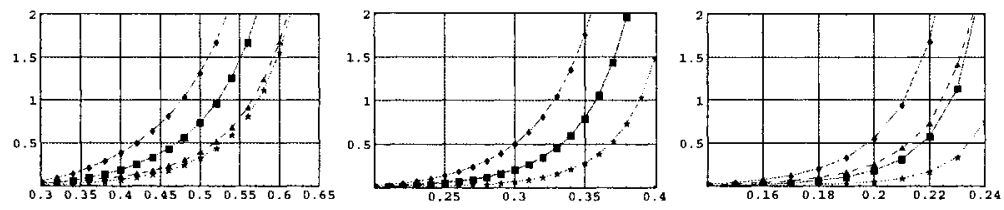

Figure 5. Mean connectivity period length for $n=20,100,500$ nodes (from left to right). Dashed curves corresponds to simulated results and solid curves to estimates.

markers correspond to our approximation where we have assumed a uniform location for isolated node. Lines with star markers correspond to our approximation with $\bar{T}_{d} \approx \hat{T}_{d}^{(0)}$, i.e., that a node becomes most likely disconnected in the center. Lines with diamond markers correspond to our approximation with $\vec{T}_{d} \approx \hat{T}_{d}^{(1)}$, i.e., that a node becomes most likely disconnected on the border. The results show that in the interesting region, where connectivity probability rises steeply, using $\bar{T}_{d} \approx \hat{T}_{d}^{(0)}$ and $\bar{T}_{d} \approx \hat{T}_{d}^{(1)}$ act as if they were lower and upper bounds for the mean connectivity durations, while the approximation with uniform assumption for the isolated node gives rather accurate results.

Finally we study how the velocity distribution affects the mean length of the connectivity period. Note that as the quantity $\lambda(r, d)$ is inversely proportional to quantity $\mathrm{E}[1 / v]$, our approximation (14) is directly proportional to quantity $\mathrm{E}[1 / v]$. In Fig. 6 the simulation results with three different velocity distributions are illustrated for $n=20,100,500$ nodes, i) $v=1$ (i.e., constant), ii) $v \sim \mathrm{U}(0.1,1.9)$ (i.e., $\bar{v}=1$ ), and iii) $v \sim \mathrm{U}(0.356,2.156)$ (i.e., $\mathrm{E}[1 / v] \approx 1$ ). Diamond markers correspond to i), star markers to ii), and square markers to iii). It can be seen that with $n=20,100,500$ nodes i) and iii) are almost identical, while ii) generally leads to longer connectivity durations. Also note that the relative difference in the results for ii) and cases i,iii) is close to $\mathrm{E}[1 / v] \approx 1.64$, in agreement with our approach.

\section{Conclusions}

In this paper, we have studied the connectivity properties of ad hoc networks where the nodes move according to the RWP mobility model. Analytical approximations have been given for estimating the probability that a network consisting of $n$ nodes is $k$-connected based on estimating the probability that the network has minimum degree $k$. The approximations were validated by numerical simulations showing remarkably good agreement.

Additionally, we have also studied the mean lengths of the connectivity periods, for which we have given an (parameterized) approximation, which utilizes the results on the arrival rate of the RWP process into a given subset area. The 

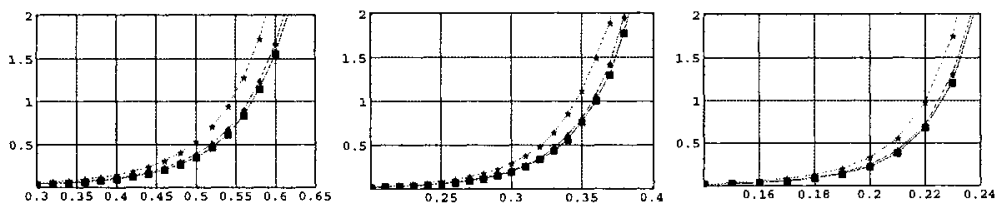

Figure 6. Mean connectivity period length for $n=20,100,500$ nodes (from left to right) with different velocity distributions.

numerical results show that in the interesting region where connectivity probability rises steeply, approximation works fairly well. Furthermore, according to our approach the mean length of the connectivity period is directly proportional to quantity $\mathrm{E}[1 / v]$, which matches well with the numerical experiments.

\section{References}

[1] C. Bettstetter, G. Resta, and P. Santi, "The node distribution of the random waypoint mobility model for wireless ad hoc networks," IEEE Trans. on Mobile Computing, vol. 2, no. 1, pp. 25-39, 2003.

[2] J. Yoon, M. Liu, and B. Noble, "Random waypoint considered harmful," in Proceedings of IEEE INFOCOM, San Fransisco, California, USA, April 2003, pp. 1312-1321.

[3] W. Navidi and T. Camp, "Stationary distributions for the random waypoint mobility model," IEEE Trans. on Mobile Computing, vol. 3, no. 1, pp. 99-108, 2004.

[4] Esa Hyytiä, Pasi Lassila, and Jorma Virtamo, "Spatial node distribution in the random waypoint mobility model with applications," submitted for publication, available at http: //www.netlab.tkk.fi/julkaisut/bib/, Mar. 2005.

[5] Esa Hyytiä and Jorma Virtamo, "Random waypoint mobility model in cellular networks," ACM/Kluwer Wireless Networks, 2005, to appear.

[6] M. Sánchez, P. Manzoni, and Z. J. Haas, "Determination of critical transmission range in Ad-Hoc networks," in Proceedings of Multiaccess Mobility and Teletraffic for Wireless Communications 1999 Workshop (MMT'99), Oct. 1999.

[7] M. D. Penrose, "The longest edge of the random minimal spanning tree," Annals of Applied Probability, vol. 7, no. 2, pp. 340-361, 1997.

[8] M. D. Penrose, "On $k$-connectivity for a geometric random graph," Random Structures and Algorithms, vol. 15, no. 2, pp. 145-164, 1999.

[9] M. D. Penrose, "Extremes for the minimal spanning tree on normally distributed points," Advances in Applied Probability, vol. 30, no. 3, pp. 628-639, 1998.

[10] Peng-Jun Wan and Chih-Wei Yi, "Asymptotic critical transmission radius and critical neighbor number for $k$-connectivity in wireless ad hoc networks," in Proceedings of $A C M$ MobiHoc '04. 2004, pp. 1-8, ACM Press.

[11] Christian Bettstetter, "On the connectivity of Ad Hoc networks," Computer Journal, vol. 47, no. 4, pp. 432-447, July 2004.

[12] H. Koskinen, "A simulation-based method for predicting connectivity in wireless multihop networks," Telecommunication Systems, vol. 26, no. 2-4, pp. 321-338, June 2004. 